\title{
Identification and characterization of the biosynthetic gene cluster of polyoxypeptin A, a potent apoptosis inducer
}

Yanhua Du', Yemin Wang ${ }^{1}$, Tingting Huang ${ }^{2}$, Meifeng Tao ${ }^{1}$, Zixin Deng ${ }^{1}$ and Shuangjun Lin ${ }^{1 *}$

\begin{abstract}
Background: Polyoxypeptin A was isolated from a culture broth of Streptomyces sp. MK498-98 F14, which has a potent apoptosis-inducing activity towards human pancreatic carcinoma AsPC-1 cells. Structurally, polyoxypeptin A is composed of a $C_{15}$ acyl side chain and a nineteen-membered cyclodepsipeptide core that consists of six unusual nonproteinogenic amino acid residues (N-hydroxyvaline, 3-hydroxy-3-methylproline, 5-hydroxypiperazic acid, $\mathrm{N}$-hydroxyalanine, piperazic acid, and 3-hydroxyleucine) at high oxidation states.

Results: A gene cluster containing 37 open reading frames (ORFs) has been sequenced and analyzed for the biosynthesis of polyoxypeptin A. We constructed 12 specific gene inactivation mutants, most of which abolished the production of polyoxypeptin $\mathrm{A}$ and only $\Delta p / y M$ mutant accumulated a dehydroxylated analogue polyoxypeptin B. Based on bioinformatics analysis and genetic data, we proposed the biosynthetic pathway of polyoxypeptin A and biosynthetic models of six unusual amino acid building blocks and a PKS extender unit.

Conclusions: The identified gene cluster and proposed pathway for the biosynthesis of polyoxypeptin A will pave a way to understand the biosynthetic mechanism of the azinothricin family natural products and provide opportunities to apply combinatorial biosynthesis strategy to create more useful compounds.
\end{abstract}

Keywords: Biosynthesis, Polyoxypeptin A, Polyketide, Nonribosomal peptide, Apoptosis-inducing activity

\section{Background}

Polyoxypeptin A (PLYA) was isolated from the culture broth of Streptomyces sp. MK498-98 F14, along with a deoxy derivative named as polyoxypeptin B (PLYB), as a result of screening microbial culture extracts for apoptosis inducer of the human pancreatic adenocarcinoma AsPC-1 cells that are highly apoptosis-resistant [1,2]. PLYA is composed of an acyl side chain and a cyclic hexadepsipeptide core that features two piperazic acid units (Figure 1). Structurally similar compounds have been identified from actinomycetes including A83586C [3], aurantimycins [4], azinothricin [5], citropeptin [6], diperamycin [7], kettapeptin [8], IC101 [9], L-156,602 [10], pipalamycin [11], and variapeptin [12] (Figure 1).

\footnotetext{
*Correspondence: linsj@sjtu.edu.cn

${ }^{1}$ State Key Laboratory of Microbial Metabolism, School of Life Sciences and Biotechnology, Shanghai Jiao Tong University, 800 Dongchuan Road, Shanghai 200240, China

Full list of author information is available at the end of the article
}

This group of secondary metabolites was named 'azinothricin family' after the identification of azinothricin as the first member in 1986 from Streptomyces sp. X-1950.

The compounds in this family exhibit diverse biological activities, such as potent antibacterial, antitumor $[13,14]$, and anti-inflammatory activities [15], and acceleration of wound healing [16]. Both PLYA and PLYB were confirmed to be potent inducers of apoptosis. They can inhibit the proliferation of apoptosis-resistant AsPC-1 cells with $\mathrm{IC}_{50}$ values of 0.062 and $0.015 \mu \mathrm{g} / \mathrm{mL}$. They can also induce early cell death in human pancreatic adenocarcinoma AsPC- 1 cell lines with $\mathrm{ED}_{50}$ values of 0.08 and $0.17 \mu \mathrm{g} / \mathrm{mL}$, more efficiently than adriamycin and vinblastine that can't induce death of AsPC-1 cells even at $30 \mu \mathrm{g} / \mathrm{mL}$ [2]. In addition, they are able to induce apoptotic morphology and internucleosomal DNA fragmentation in AsPC-1 cell lines at low concentrations [17].

Polyoxypeptins (A and B) possess a variety of attractive biosynthetic features in their structures. The $\mathrm{C}_{15}$ acyl side chain may present a unique extension unit in polyketide 


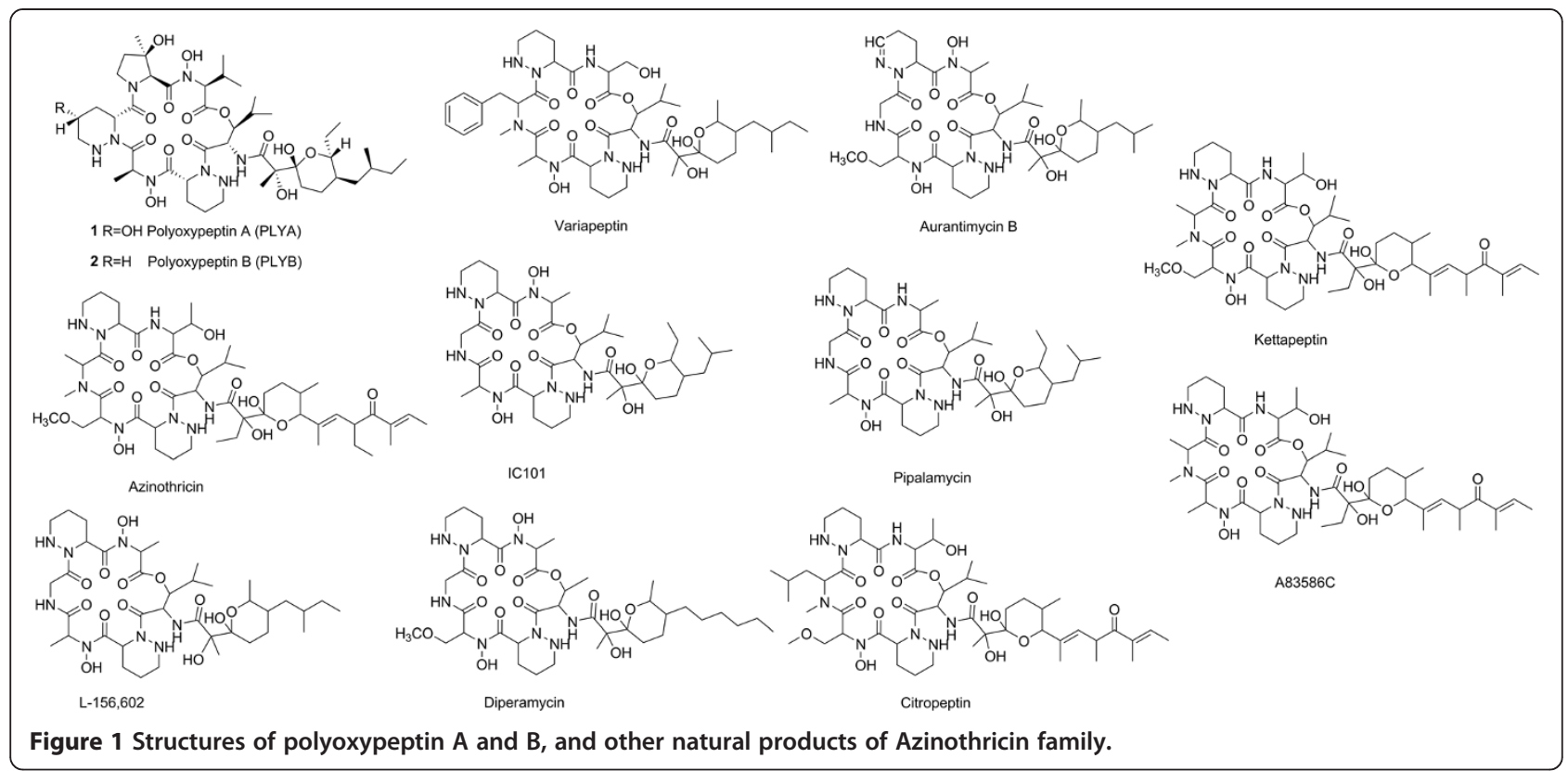

synthase (PKS) assembly line probably derived from isoleucine [18]. The cyclo-depsipeptide core consists of six unusual amino acid residues at high oxidation states, including 3-hydroxyleucine, piperazic acid, Nhydroxyalanine, 5-hydroxypiperazic acid (for PLYA) or piperazic acid (for PLYB), 3-hydroxy - 3-methylproline, and $\mathrm{N}$-hydroxyvaline. The most intriguing is the hydroxylation at $\alpha$-amino groups of the $\mathrm{L}$-alanine and $\mathrm{L}$-valine, different from that at terminal amino group of ornithine or lysine in siderophore biosynthesis [19]. It is worth to note that $(2 S, 3 R)$-3-hydroxy - 3-methylproline presents a synthetic challenge [20]. Both structural novelty and biological activity of polyoxypeptins have spurred much interest in understanding the biosynthetic mechanism and employing biosynthesis and combinatorial biosynthesis to create new polyoxypeptin derives.

Here, we report the identification and characterization of the biosynthetic gene cluster for PLYA based on the genome sequencing, bioinformatics analysis, and systematic gene disruptions. The five stand-alone nonribosomal peptide synthetase (NRPS) domains were confirmed to be essential for PLYA biosynthesis, putatively involved in the biosynthesis of the unusual building blocks for assembly of the peptide backbone. Furthermore, three hydroxylases and two P450 enzymes were genetically characterized to be involved in the biosynthesis of PLYA. Among them, the P450 enzyme PlyM may play a role in transforming PLYB to PLYA.

\section{Results and discussion}

Identification and analysis of the ply gene cluster Whole genome sequencing of Streptomyces sp. MK49898 F14 using the 454 sequencing technology yielded
$11,068,848$ bp DNA sequence spanning 528 contigs. Based on the structural analysis of PLYs, we hypothesized that PLYs are assembled by a hybrid PKS/NRPS system. Bioinformatics analysis of the whole genome revealed at least 20 NRPS genes and 70 PKS genes. Among them, the contig00355 (48439 bp DNA sequence) attracted our attention because it contains 7 putative NRPS genes and 4 PKS genes encoding total 4 PKS modules that perfectly match the assembly of the $C_{15}$ acyl side chain based on the colinearity hypothesis [21]. Moreover, orf14777 ( $p l y P)$ annotated as an L-proline-3-hydroxylase may be involved in the hydroxylation of 3-methylproline, one of the proposed precursor of PLYA [18]. NRPS analysis program revealed that 7 NRPS genes encode a free-standing peptidyl carrier protein (PCP) (PlyQ), 3 stand-alone thioesterase (TE) domains (PlyI, PlyS, and PlyY), and 3 NRPS modules that are not sufficient for assembly of the hexapeptide. Therefore, we continued to find another relevant contig00067 (83207 bp DNA sequence) contains 4 NRPS genes encoding a free-standing adenylation (A) domain (PlyC) and PCP (PlyD), and 3 NRPS modules. Taken together, the total 6 NRPS modules and 4 PKS modules are sufficient for the assembly of PLYs.

To confirm involvement of the genes in these two contigs by disruption of specific NRPS genes, a genomic library of Streptomyces sp. MK498-98 F14 was constructed using SuperCos1 [22] and 23000 clones were obtained. Two pairs of primers (Additional file 1: Table S3) were designed on the base of two hydroxylases (PlyE and PlyP) from the contig00067 and contig00355, respectively, and used to screen the cosmid library using PCR method [23]. 10 positive cosmids derived from the 


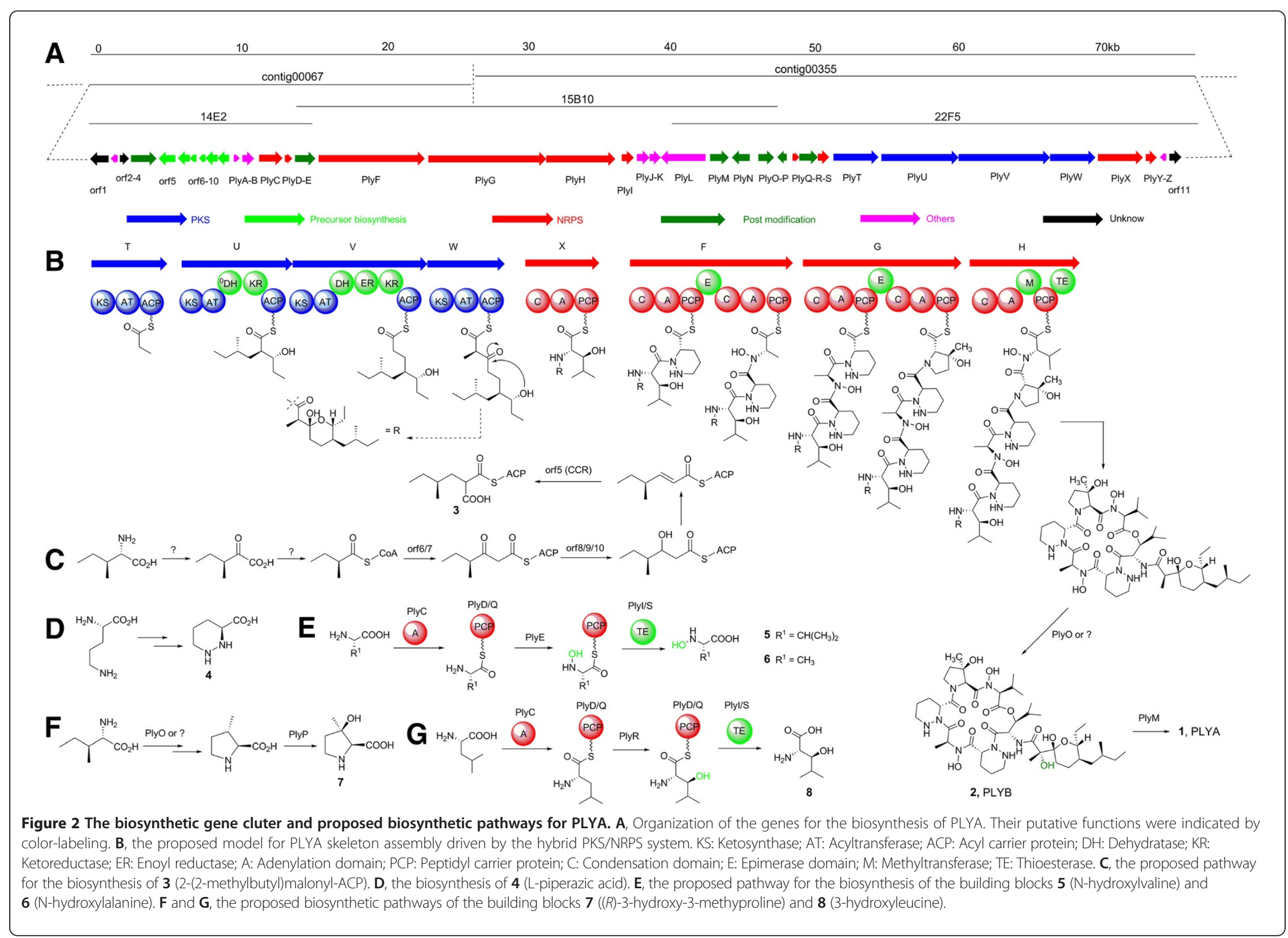


primer of $p l y E$ and 11 positive cosmids derived from the primer of $p l y P$ were obtained. Interestingly, these two sets of cosmids overlapped one same cosmid, 15B10, which gave the further evidence that these two contigs belong to the same contig (Figure 2A). Thus, we used $15 \mathrm{~B} 10$ as a template to fill the gap between these two contigs by PCR sequencing and got a 131,646 bp contiguous DNA sequence (Figure 2A). Subsequently, a NRPS gene orf14800 $(p l y H)$ was inactivated by replacement of plyH with apramycin resistant gene $(\operatorname{aac}(3) I V$-oriT) cassette in the genome of Streptomyces sp. MK498-98 F14 (Additional file 1: Scheme S1). The resulting doublecrossover mutant completely abolished the production of PLYA (Figure 3, trace i), confirming that the genes in this region are responsible for biosynthesis of PLYs.

Bioinformatics analysis suggested that 37 open reading frames (ORFs, Figure 2A and Table 1) spanning $75 \mathrm{~kb}$ in this region were proposed to constitute the ply gene cluster based on the functional assignment of the deduced gene products. Among them, 4 modular type I PKS genes (plyTUVW) and 4 modular NRPS genes (plyXFGH) encoding 4 PKS modules and 6 NRPS modules are present for the assembly of the PLY core structure (Figure 2B). Other 6 NRPS genes (plyCDQISY) encode an A domain, two PCPs, and three TEs that are free-standing from the modular NRPSs. They are suggested to be involved in the biosynthesis of nonproteinogenic amino acid building blocks. 6 genes (orf5-orf10) are proposed to be involved in the biosynthesis of a

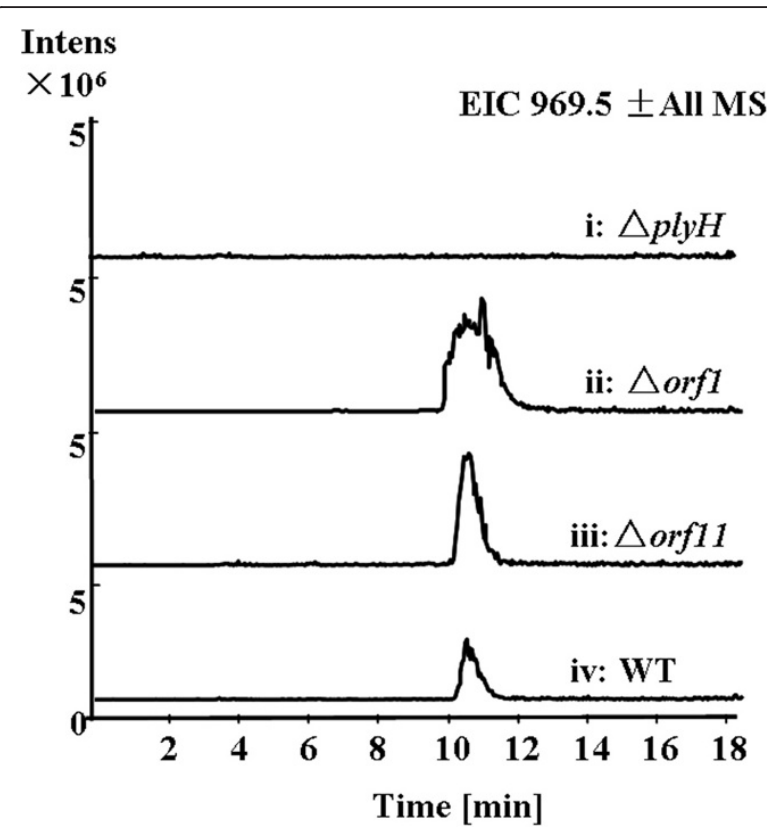

Figure 3 Verification of the ply gene cluster. LC-MS analysis (extracted ion chromatograms of $\mathrm{m} / \mathrm{z}[\mathrm{M}+\mathrm{H}]^{+} 969.5$ corresponding to PLYA) of Streptomyces sp. MK498-98 F14 wild type (indicated with WT) and mutants ( $\Delta$ orf1, $\Delta$ orf1 1, and $\Delta p / y H)$. novel extender unit for PKS assembly (Figure 2C). There are 6 genes (orf4 and plyEMOPR) encoding putative hydroxylases or oxygenases that are proposed to responsible for the biosynthesis of unusual building blocks or post-modifications (Figure 2D-G). There are $2 \mathrm{ABC}$ transporter genes ( $p l y J$ and $p l y K)$ and 4 putative regulatory genes (orf $2, p l y B, p l y L$, and $p l y Z)$. In addition, an aminotransferase gene $(p l y N)$ is located in the center of the ply gene cluster that is probably involved in the biosynthesis of the novel PKS extender unit (3) (Figure 2C).

Upstream of the ply gene cluster, three genes, orf03394 (orf1), orf03396 and orf03399, encoding proteins with similarities to 3-dehydroquinate synthase, sugar kinase and nucleotidyl transferase respectively, seemingly have no relationship with the biosynthesis of PLYA. orf03392 (orf2), adjacent to orf1, is predicted to encode a protein with similarity to a transcriptional regulator, which may be involved in the biosynthesis of PLYs. Downstream of the ply gene cluster, three genes, orf14746 (plyZ), orf14744 (orf11) and orf14742 encode proteins with similarities to LysR family transcriptional regulator, hypothetical protein ROP_29250 and hypothetical protein ROP_03220. To prove that the genes beyond this cluster are not related to PLY biosynthesis, we inactivated orf1 and orf11. The resulting mutants have no effect on the PLYA production (Figure 3, trace ii and iii), indicating that the 37 ORFs-contained ply gene cluster is responsible for the PLYs biosynthesis.

\section{Assembly of the $\mathrm{C}_{15}$ acyl side chain by PKSs}

Within the ply cluster, 4 modular type I PKS genes (plyTUVW) encode four PKS modules, the organization of which is accordant with the assembly of the $C_{15}$ acyl side chain of PLYA via three steps of elongation from the propionate starter unit (Figure 2B). Both PlyT and PlyW consist of ketosynthase (KS), acyltransferase (AT), and acyl carrier protein (ACP). However, the active site Cys (for transthioesterification) of the PlyT-KS is replaced with Gln (Additional file 1: Figure S1), so it belongs to the so called " $\mathrm{KS} \mathrm{Q}_{\mathrm{Q}}$ " that often occurs in the loading module of PKS system [24]. Therefore, PlyT acts as a loading module for formation of the propionate starter unit by catalyzing decarboxylation of methylmalonyl group after tethering onto ACP (Figure 2B). The conserved regions of AT domain including the active site motif GHSQG [25] in both PlyT and PlyW (Additional file 1: Figure S2), along with substrate specificity code (YASH) [26] indicate that both ATs are specific for methylmalonyl-CoA, consistent with the structure of the side chain of PLYA (Figure 2B). In PlyU, in addition to $\mathrm{KS}, \mathrm{AT}$, and ACP domains, a dehydratase (DH) domain and a ketoreductase (KR) domain are present. However, the DH domain here is believed to be nonfunctional because the key amino acid residue $\mathrm{H}$ of the 
Table 1 Deduced functions of ORFs in the biosynthetic gene cluster of PLYA

\begin{tabular}{|c|c|c|c|c|c|}
\hline Gene & Size $^{a}$ & Accession no. & Proposed function & Homologous protein species & Identity/Similarity \\
\hline orf03399 & 384 & YP_003099796 & Nucleotidyl transferase & Actinosynnema mirum DSM 43827 & $64 / 73$ \\
\hline orf03396 & 309 & YP_004903951 & putative sugar kinase & Kitasatospora setae KM-6054 & $50 / 62$ \\
\hline orf1 & 422 & YP_003099794 & 3-dehydroquinate synthase & Actinosynnema mirum DSM 43827 & $56 / 69$ \\
\hline orf2 & 128 & EID72461 & MarR family transcriptional regulator & Rhodococcus imtechensis RKJ300 & $71 / 83$ \\
\hline orf3 & 146 & ZP_09957194 & Hypothetical protein & Streptomyces chartreusis NRRL 12338 & $75 / 84$ \\
\hline orf4 & 566 & CAJ61212 & Putative polyketide oxygenase/hydroxylase & Frankia alni ACN14a & $77 / 83$ \\
\hline orf5 & 377 & ZP_04706918 & Alcohol dehydrogenase BadC & Streptomyces roseosporus NRRL 11379 & $76 / 86$ \\
\hline orf6 & 312 & ZP_06582592 & 3-oxoacyl-[acyl-carrier-protein] synthase III & Streptomyces roseosporus NRRL 15998 & $71 / 82$ \\
\hline orf7 & 82 & ZP_04706920 & Hypothetical protein & Streptomyces roseosporus NRRL 11379 & $59 / 75$ \\
\hline orf8 & 82 & ZP_04706921 & Dihydrolipoamide succinyltransferase & Streptomyces roseosporus NRRL 11379 & $65 / 81$ \\
\hline orf9 & 326 & ZP_06582595 & 2-oxoisovalerate dehydrogenase & Streptomyces roseosporus NRRL 15998 & $75 / 87$ \\
\hline orf10 & 303 & ZP_04706923 & Pyruvate dehydrogenase & Streptomyces roseosporus NRRL 11379 & $74 / 84$ \\
\hline plyA & 71 & YP_640626 & MbtH-like protein & Mycobacterium sp. MCS & $80 / 87$ \\
\hline plyB & 225 & YP_712760 & Putative regulator & Frankia alni ACN14a & $76 / 84$ \\
\hline plyc & 528 & YP_712761 & A & Frankia alni ACN14a & $77 / 85$ \\
\hline plyD & 77 & YP_712762 & PCP & Frankia alni ACN14a & $85 / 94$ \\
\hline plyE & 395 & YP_712763 & Putative hydroxylase & Frankia alni ACN14a & $76 / 86$ \\
\hline plyF & 2583 & ABV56588 & C-A-PCP-E-C-A-PCP & Kutzneria sp. 744 & $56 / 68$ \\
\hline plyG & 2809 & ZP_05519638 & C-A-PCP-E-C-A-PCP & Streptomyces hygroscopicus ATCC 53653 & $73 / 82$ \\
\hline plyH & 1662 & BAH04161 & C-A-M-PCP-TE & Streptomyces triostinicus & $72 / 82$ \\
\hline plyl & 247 & YP_712767 & TE & Frankia alni ACN14a & $80 / 87$ \\
\hline ply」 & 312 & YP_003112824 & Daunorubicin resistance $A B C$ transporter & Catenulispora acidiphila DSM 44928 & $78 / 90$ \\
\hline plyk & 253 & YP_712769 & $\mathrm{ABC}$ transporter system & Frankia alni ACN14a & $71 / 81$ \\
\hline plyl & 1043 & YP_003112826 & Transcriptional regulator & Catenulispora acidiphila DSM 44928 & $72 / 80$ \\
\hline plyM & 412 & AAT45271 & Cytochrome P450 monooxygenase & Streptomyces tubercidicus & $43 / 59$ \\
\hline plyN & 450 & ZP_04604097 & Aminotransferase class I and II & Micromonospora sp. ATCC 39149 & $58 / 70$ \\
\hline plyO & 308 & ZP_03862696 & Polyketide fumonisin & Kribbella flavida DSM 17836 & $48 / 64$ \\
\hline plyP & 270 & ZP_04292518 & L-proline 3-hydroxylase type II & Bacillus cereus R309803 & $32 / 51$ \\
\hline plyQ & 88 & YP_712776 & PCP & Frankia alni ACN14a & $76 / 89$ \\
\hline plyR & 415 & YP_712777 & Cytochrome P450 monooxygenase & Frankia alni ACN14a & $77 / 85$ \\
\hline plys & 245 & AAT45287 & TE & Streptomyces tubercidicus & $70 / 81$ \\
\hline plyT & 1031 & YP_712779 & KS-AT-ACP & Frankia alni ACN14a & $71 / 80$ \\
\hline plyU & 1872 & YP_712780 & KS-AT-DH-KR-ACP & Frankia alni ACN14a & $69 / 79$ \\
\hline plyV & 2199 & YP_712781 & KS-AT-DH-ER-KR-ACP & Frankia alni ACN14a & $70 / 78$ \\
\hline plyW & 1041 & YP_712782 & KS-AT-ACP & Frankia alni ACN14a & $72 / 82$ \\
\hline plyx & 1080 & YP_712783 & C-A-PCP & Frankia alni ACN14a & $62 / 72$ \\
\hline plyy & 253 & ZP_04472110 & TE & Streptosporangium roseum DSM 43021 & $70 / 78$ \\
\hline plyz & 79 & YP_001612061 & LysR family transcriptional regulator & Sorangium cellulosum & $65 / 77$ \\
\hline orf11 & 287 & YP_702564 & Hypothetical protein & Rhodococcus jostii RHA1 & $56 / 73$ \\
\hline orf14742 & 170 & YP_002777514 & Hypothetical protein ROP_03220 & Rhodococcus opacus B4 & $51 / 63$ \\
\hline
\end{tabular}

${ }^{\mathrm{a}}$ Numbers are in amino acids.

conserved motif HxxxGxxxxP [27] is replaced by Gln (Additional file 1: Figure S3). The conserved motif of PlyU-AT for substrate selectivity is VPGH, neither including the serine residue in YASH for methylmalonylCoA nor phenylalanine residue in HAFH for malonylCoA (Additional file 1: Figure S2). These changes may 
broaden the substrate binding pocket and enhance hydrophobicity of the substrate binding pocket, supporting that $\mathrm{PlyU}$ is able to recognize 2-(2-methylbutyl)malonyl 3 as an unusual extender unit (Figure 2C). Compared to PlyU, PlyV contains an active DH domain and an enoyl reductase (ER) domain. The conserved motif (HAFH) of PlyVAT signifies it specific for malonyl-CoA as the extender unit (Figure 2B and Additional file 1: Figure S2). Taken together, PlyTUVW seem to be sufficient for the assembly of the $\mathrm{C}_{15}$ acyl side chain of PLYA.

\section{Biosynthesis of 2-(2-methylbutyl)malonyl extender unit 3}

The structural analysis of PLYs and PKS architecture suggest that an unusual PKS extender unit 2-(2-methylbutyl)malonyl-CoA (or ACP, 3) is required for the assembly of the $C_{15}$ acyl side chain of PLYs. The biosynthesis of the 2-(2-methylbutyl)malonyl-CoA (or ACP) extender unit 3 would involve a reductive carboxylation mediated by a crotonyl-CoA reductase/carboxylase (CCR) homolog. Similar reactions have been reported for formation of ethylmalony-CoA [28,29], 2-(2-chloroethyl)malonyl-CoA [30], and hexylmalonyl-CoA [31], as well as proposed for involvement of biosynthesis of cinnabaramides [32], thuggacins [33], sanglifehrins [34], germicidins and divergolides [35], ansalactams [36] and many other natural products. Analysis of the ply cluster reveals orf5 encoding a CCR TgaD homolog (identity/similarity, 46\%/59\%) that was proposed to be involved in the biosynthesis of hexylmalonyl-CoA, an extender unit for the assembly of thuggacin [33]. orf6, adjacent to orf5, encodes a protein shared $71 \%$ identity and $81 \%$ similarity with 3-oxoacylACP synthase III from $S$. roseosporus NRRL 15998. The gene orf7, located upstream of orf6, encodes an ACP that contains a catalytic motif DLDLDSL (the Serine is for phosphopantethein modification) [24]. The presence of these two genes indicates that the extender unit 2(2-methylbutyl)malonyl may be tethered to ACP, not to $\mathrm{CoA}$. In study of the biosynthesis of isobutylmalonyl-CoA extender unit for germicidins and divergolides, CCR, KSIII and $\mathrm{HBDH}$ (a 3-hydroxybutyryl-CoA hydrogenase) are transcribed in the same operon [35]. orf567 and other three genes orf8910 also constitute an operon (Figure 2A). The genes orf8910 encode $\alpha$-keto acid dehydrogenase E2 component, E1 component $\beta$ and $\alpha$ subunits, respectively, suggesting their involvement of the biosynthesis of $\mathbf{3}$ by reduction of the $\beta$-keto group (Figure $2 \mathrm{C}$ ). Given that the previous feeding study with isotope-labeled precursor suggested this 2-(2-methylbutyl)malonyl unit derived from isoleucine via a transamination [18], we proposed that an aminotransferase is required for the formation of $\alpha$-keto acid, as shown in Figure 2C. $p l y N$ is the only identified aminotransferase gene, so we constructed the $\triangle p l y N$ mutant by replacement of the $p l y N$ gene with the $\operatorname{aac}(3) I V$-oriT cassette (Additional file 1: Scheme S2).
However, $\Delta p l y N$ was found no effect on the PLYA production (Figure 4, trace viii), so we assume that other aminotransferases may mediate this transamination for the incorporation of $\mathrm{C}_{5}$ unit of isoleucine into 3 (Figure 2C).

\section{Assembly of the cyclodepsipeptide by NRPSs}

After the $\mathrm{C}_{15}$ acyl side chain is assembled by 4 modular PKSs, it is transferred to 3-hydroxyleucine via an amide bond formation catalyzed by a NRPS, thus initiating the assembly of the peptide core. Within the biosynthetic gene cluster, there are 4 genes plyFGHX encoding modular NRPS proteins. Both PlyF and PlyG consist of two modules with seven domains (C-A1-PCP-E-C-A2-PCP) (Figure 2B). Active epimerase (E) domains are present indicating that the amino acids activated by PlyF-A1 and PlyG-A1 should be converted into D-configuration.

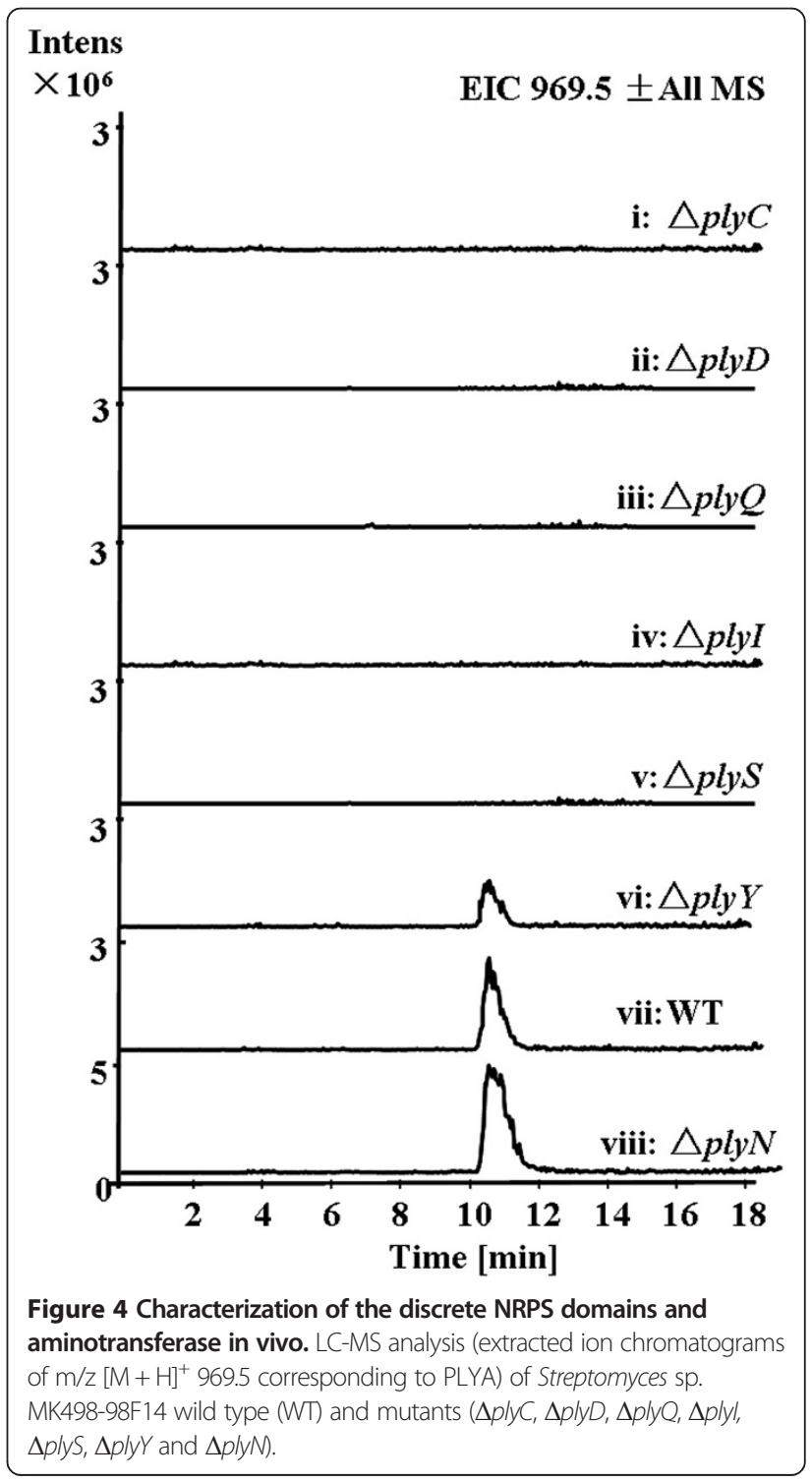


Among the six nonproteinogenic amino acid residues, only two piperazic acid residues are $\mathrm{D}$-configuration, so these two A domains (PlyF-A1 and PlyG-A1) are proposed to recognize and activate L-piperazic acid (4, Figure 2D) that was confirmed to be derived from Lornithine [37]. This assumption can be supported by the findings that PlyF-A1 shares $52-59 \%$ identity and $64-69 \%$ similarity to PlyG-A1, KtzH-A1 [38], and HmtL-A1 [39] (Additional file 1: Figure S4), and as well as the substrate specificity-conferring ten amino acids (DVFSVASYAK for PlyF-A1 and DVFSIAAYAK for PlyG-A1) are highly analogous to those of KtzH-A1 (DVFSVGPYAK) and HmtL-A1 (DVFSVAAYAK) [40,41]. Both KtzH-A1 and HmtL-A1 were proposed to recognize and activate Lpiperazic acid $[38,39]$. PlyH contains five domains (C-AM-PCP-TE) with a thioesterase (TE) domain present, indicating that $\mathrm{PlyH}$ is the last module of PLY NRPS system and responsible for the release and cyclization of the peptide chain via an ester bond formation. It is striking that an active methyltransferase (M) domain (containing the SAM-binding sites EXGXGXG) is present in the PlyH [42], but no N-methyl group is present in the structure of PLYs. The presence of this M domain remains enigmatic. Based on the PLY structure analysis and NRPS machinery [43], PlyH-A is proposed to recognize N-hydroxyvaline (5, Figure $2 \mathrm{E}$ ) as its substrate, but not valine because its substrate specificity-conferring codon sequences (DAPFEALVEX) are significantly distinct from those found for valine-specificity (DALWMGGTFK) [44]. Subsequently, the whole sequence of PlyH-A shows $76 \%$ identity and $83 \%$ similarity to that of PlyF-A2, indicating that PlyF-A2 is specific for N-hydroxyalanine (6, Figure 2E and Additional file 1: Figure S5). These assignments are consistent with the amino acid sequence of the peptide core of PLYs. Finally, according to the collinearity of the NRPS modules and the building blocks of the NRPS-derived products, PlyG-A2 and PlyX would be proposed to recognize and activate $(R)$-3-hydroxy-3methylproline (7, Figure 2F) and 3-hydroxyleucine (8. Figure 2G), respectively, although we can't predict their substrates based on their substrate specificity codons (Additional file 1: Table S4). Taken together, six NRPS modules activate six non-natural amino acids, and the substrate recognized by each domain is exactly consistent with the structure of the cyclic depsipeptide of PLYs (Figure 2B).

\section{Biosynthesis of nonproteinogenic amino acid building blocks}

Except for the modular NRPSs, there are six discrete NRPS genes present in the ply gene cluster (Table 1 and Figure 2A), identified as an A domain (PlyC), two PCP domains (PlyD, PlyQ) and three TE domains (PlyI, PlyS, PlyY). To test whether these six free-standing domains were involved in the biosynthesis of PLYA, we constructed their disruption mutants by gene replacement with the aac(3)IV-oriT cassette (Additional file 1: Scheme S3-8). The mutant strains $(\Delta p l y C, \Delta p l y D, \Delta p l y Q, \Delta p l y I$ and $\triangle p l y S)$ completely abolished the production of PLYA (Figure 4, traces i-v), indicating that these 5 discrete NRPS domains are essential for the PLYA biosynthesis. However, the $\Delta p l y Y$ mutant strain still produced PLYA, but the productivity decreased in comparison with that of the wild type strain (Figure 4, trace vi and vii). Therefore, PlyY may act as a type II TE, probably playing an editing role in the biosynthesis of PLYA by hydrolyzing misincorporated building blocks. Multiple sequence alignment reveals that PlyY and typical type II TEs contain a conserved motif (GHSXG) and catalytic triad S/C-D-H that is consistent with hydrolytic function (Additional file 1: Figure S6) [45-47]. This catalytic triad is also present in PlyI and PlyS, indicating the hydrolytic function of PlyI and PlyS, as shown by Figure 2E and G.

The discrete NRPS domains have been found in many NRPS assembly lines responsible for the formation of nonproteinogenic building blocks [21,48]. For example, the conversion of proline to pyrrole-2-carboxylic acid, which is a precursor for the biosynthesis of pyoluteorin, prodigiosin, and clorobiocin [49], occurs while proline is activated by a discrete A domain and covalently tethered in a thioester linkage to a T domain. Since all the A domains of six modular NRPSs in the PLY biosynthetic pathway are proposed to recognize and activate nonproteinogenic amino acid building blocks, PlyCDQIS are assumed to be responsible for the formation of several monomers of PLYs from the natural amino acids. Given that we can't predict the substrate based on the key residues of the substrate-binding pocket of PlyC (A domain), we propose that $\mathrm{PlyC}$ may activate multiple amino acids such as alanine and valine or leucine, and tether them to the corresponding PCPs (PlyD and PlyQ). After Nhydroxylation of alanine and valine (Figure $2 \mathrm{E}$ ) as well as $\beta$-hydroxylation of leucine (Figure 2G), the matured building blocks are proposed to be released by discrete TEs (PlyI or PlyS, respectively) and activated again by PlyF-A2, $\mathrm{PlyH}$, and PlyX, respectively (Figure $2 \mathrm{~B}$ ). Such processes are rare events in typical NRPS-driven biosynthetic pathways [21].

The depsipeptide core of PLYA is composed of 6 amino acids, 5 of which are hydroxylated. There are 6 genes encoding putative hydroxylases or oxygenases. For example, plyR encodes a cytochrome P450 monooxygenase that shows high homology (37\% identity and 54\% similarity) to NikQ that was demonstrated to catalyze $\beta$ hydroxylation of histidine tethered to PCP, so we could propose that PlyR may be involved in the formation of $\beta$-hydroxyleucine building block (Figure 2G). Indeed, inactivation of $p l y R$ resulted in loss of ability to produce 
PLYA (Figure 5A, trace i). Given that FAD-dependent monooxygenase $\mathrm{CchB}$ has been reported to catalyze the $\mathrm{N}$-hydroxylation of the $\delta$-amino group of ornithine in the biosynthetic pathway of the siderophore coelichelin [50], we proposed that PlyE, a FAD-dependent monooxygenase, may be responsible for $\mathrm{N}$-hydroxylation of alanine and valine when they are activated and tethered to a PCP by A domain PlyC (Figure 2E). The $\triangle p l y E$ mutant lost ability to produce PLYA (Figure 5A, trace ii), indicating its possible role in formation of $\mathrm{N}$-hydroxyalanine and $\mathrm{N}$ hydroxyvaline. PlyP, a L-proline 3-hydroxylase, should be responsible for hydroxylation of 3-methyl-L-proline that is biosynthesized from L-isoleucine demonstrated by isotopefeeding study (Figure 2F) [18]. Inactivation of plyP indeed abolished the production of PLYA (Figure 5A, trace iii). Recently, Tang and co-workers have reported that an $\alpha$-ketoglutarate dependent dioxygenase EcdK catalyzes a sequential oxidations of leucine to form the immediate precursor of 4-methylproline [51]. In the ply cluster, the only gene $p l y O$ encodes an $\alpha$-ketoglutarate dependent dioxygenase, but it doesn't share any homology to EcdK. In contrast, PlyO shows $48 \%$ identity and $64 \%$ similarity to phytanoyl-CoA dioxygenase (YP_003381511 from Kribbella flavida DSM 17836). It remains unclear whether $\mathrm{PlyO}$ may be responsible for the hydroxylation of the carbon adjacent to the acyl group of the $C_{15}$ acyl side chain or for the formation of 3-methyl-L-proline from L-isoleucine. orf4 encodes a FAD-binding oxygenase or hydroxylase with high homology to type II PKS-assembled aromatic compounds hydroxylase (Table 1). Its role in biosynthesis of PLYA remains unclear, but it might be involved in the biosynthesis of a building block because its inactivation abolished the PLY production (Figure 5A, trace iv).

Piperazic acid is an attractive building block of many complex secondary metabolites such as Antrimycin [52], Chloptosin [53], Himastatin [39], Luzopeptin [54], Quinoxapeptin [55], Lydiamycin [56], Piperazimycin [57] and Sanglifehrin [58]. The detailed biosynthetic mechanisms by which piperazic acid are formed are not well understood. Recently, Walsh and coworkers demonstrated that KtzI, a homolog of lysine and ornithine Nhydroxylases catalyzes the conversion of ornithine into
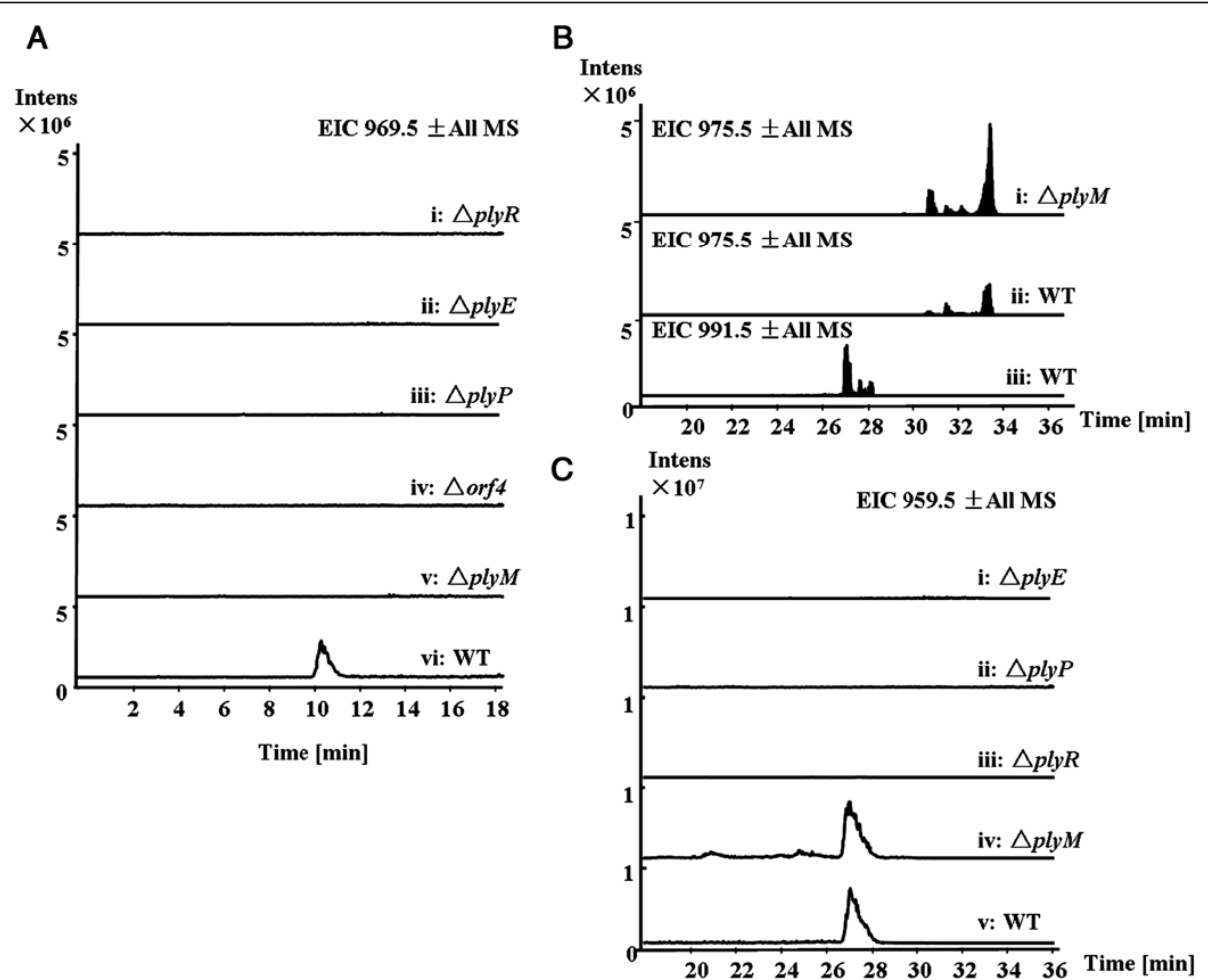

Figure $\mathbf{5}$ Characterization of the genes encoding hydroxylases or oxygenases. A, LC-MS analysis (extracted ion chromatograms of $\mathrm{m} / \mathrm{z}$ $[\mathrm{M}+\mathrm{H}]^{+} 969.5$ corresponding to PLYA) of Streptomyces sp. MK498-98F14 wild type (WT) and mutants ( $\Delta p / y E, \Delta p l y P, \Delta p l y R, \Delta$ orf4, and $\left.\Delta p l y M\right)$. B, LC-MS analysis (extracted ion chromatograms of $\mathrm{m} / \mathrm{z}[\mathrm{M}+\mathrm{Na}]^{+} 975.5$ and 991.5 corresponding to PLYB and PLYA) of Streptomyces sp. MK49898F14 wild type (WT) and the $\Delta$ plyM mutant. C, LC-MS analysis (extracted ion chromatograms of $\mathrm{m} / \mathrm{z}[\mathrm{M}+\mathrm{Na}]^{+} 959.5$ corresponding to the putative biosynthetic intermediate of PLYA lacking two hydroxyl groups) of Streptomyces sp. MK498-98F14 wild type (WT) and mutants ( $\Delta p / y E, \Delta p l y P$, $\Delta p l y R$ and $\Delta p l y M)$. B was performed under the conditions: 35-95\% B (linear gradient, 0-20 min), 100\% B (21-25 min), 35\% B (25-40 min) at the flow rate of $0.3 \mathrm{~mL} / \mathrm{min}$. 
piperazic acid in kutzneride biosynthetic pathway [37]. No such a homolog was found in the ply gene cluster, but two putative homologs are located outside the ply gene cluster (Orf11257 and Orf14738), suggesting that the biosynthesis of piperazic acid may follow the same pathway (Figure 2D).

\section{Genes putatively for post-modifications}

Most modifications in PLYA biosynthesis take place for the formation of the non-natural building blocks. Recently, Ju and co-workers demonstrated that a cytochrome P450 monooxygenase HtmN catalyzes the hydroxylation of the piperazic acid after peptide formation [59]. There are two cytochrome P450 monooxygenase genes (plyM and $p l y R$ ) in the ply cluster. PlyR was proposed to hydroxylate leucine that is tethered to a PCP, so we would assume that PlyM may catalyze the hydroxylation of piperazic acid unit as a post-modification although it doesn't show any homology to HmtN [39]. To test this hypothesis, we constructed the double-crossover mutant by replacement of $p l y M$ with the aac(3)IV-oriT gene cassette that is not producing PLYA (Figure 5A, trace v), only accumulating PLYB (Figure $5 \mathrm{~B}$ ). These findings indicate that PlyM is responsible for the conversion of PLYB into PLYA (Figure 2B). To test whether other oxygenases or hydroxylases are involved in the post-modifications, the mass corresponding to the putative intermediate of PLYA lacking two hydroxyl groups was monitored for the mutant strains (Figure 5C). This mass is only detected from the fermentation broth of wide type and $\triangle p l y M$ strains (Figure $5 \mathrm{C}$, trace $\mathrm{v}$ and iv), not from other mutant strains $(\triangle p l y E$, $\triangle p l y P$ and $\triangle p l y R$ ) indicating that the assembly of PLYA and possible intermediates is abolished. These data may support that these genes are involved in the formation of building blocks, not post-modifications. They also indicate that it is very likely to have two steps of post-hydroxylation modifications for maturation of PLYA (Figure 2B). When and how the hydroxylation at the $\alpha$-carbon of the $C_{15}$ acyl side chain takes place are still unclear.

\section{Conclusions}

We identified and characterized the ply gene cluster composed of 37 open reading frames (ORFs) by genomic sequencing and systematic gene disruptions. The biosynthetic pathway has been proposed based on bioinformatics analysis, the structural analysis of PLYs and genetic data. It was demonstrated that five discrete NRPS domains are essential for the biosynthesis of PLYs and proposed their roles in maturation of three unusual amino acid building blocks. The proposed biosynthetic pathway for PLYs will open the door to understand the biosynthesis of this family of secondary metabolites and set a stage to explore combinatorial biosynthesis to create new compounds with improved pharmaceutical properties.

\section{Ethics statement}

This study doesn't involve human subjects or materials.

\section{Methods}

Strains, plasmids, primers and culture conditions

Strains, plasmids and primers used in the study are summarized in Additional file 1: Tables S1, S2 and S3 of the supplemental material. Escherichia coli strains were cultured on Luria-Bertani (LB) broth and agar medium at $37^{\circ} \mathrm{C}$. Streptomyces sp. MK498-98 F14 and its mutant strains were cultivated at $30^{\circ} \mathrm{C}$ on the medium (yeast extract $0.4 \%$, glucose $0.4 \%$, malt extract $1 \%$, agar $1.2 \%$, $\mathrm{pH}$ 7.2) for sporulation and on 2CM [60] medium (soluble starch $1 \%$, tryptone $0.2 \%, \mathrm{NaCl} 0.1 \%,\left(\mathrm{NH}_{4}\right)_{2} \mathrm{SO}_{4}$ $0.2 \%, \mathrm{~K}_{2} \mathrm{HPO}_{4} 0.1 \%, \mathrm{MgSO}_{4} 0.1 \%, \mathrm{CaCO}_{3} 0.2 \%$, agar $1.2 \%$ with $1 \mathrm{~mL}$ inorganic salt solution per liter, $\mathrm{pH} 7.2$ ) for conjugation. For fermentation, mycelia of strain MK498-98 F14 and its mutants from the solid plates were inoculated into a 500-mL Erlenmeyer flask containing $100 \mathrm{~mL}$ of a medium composed of glucose 1\%, potato starch $1 \%$, glycerol $1 \%$, polypepton $0.5 \%$, meat extract $0.5 \%$, sodium chloride $0.5 \%$, and calcium carbonate $0.32 \%$ (adjusted to $\mathrm{pH} 7.4$ ) [2]. The culture was incubated at $28^{\circ} \mathrm{C}$ for six days on a rotatory shaker at $220 \mathrm{rpm}$.

\section{General genetic manipulations and reagents}

The general genetic manipulation in E. coli and Streptomyces were carried out following the standard protocols [22]. PCR amplifications were performed on a Veriti thermal cycler (Applied Biosystems, Carlsbad, CA) using Taq DNA polymerase. DNA fragments and PCR products were purified from agarose gels using a DNA Gel Extraction Kit (Omega). Primers were synthesized in Sangong Biotech Co. Ltd. Company (Shanghai, China). All DNA sequencing was accomplished at Shanghai Majorbio Biotech Co. Ltd (Shanghai, China). Restriction enzymes were purchased from New England Biolabs (Ipswich, MA) and Fermentas (St. Leon-Rot, Germany). Taq DNA polymerase and DNA ligase were purchased from Takara Co. Ltd. Company (Dalian, China).

\section{Genomic library construction and screening}

A genomic cosmid library of Streptomyces sp. MK49898 F14 derived from SuperCos1 was constructed according to the procedure as described by the SuperCos1 Cosmid Vector Kit. E. coli EPI300 ${ }^{\mathrm{mm}}-\mathrm{T} 1^{\mathrm{R}}$, instead of E.coli XL1-Blue MR, was used as the host strain. The total number of recombinant clones was about 3000 and then stored at $-70^{\circ} \mathrm{C}$. Two pairs of primers for two hydroxylase 
genes, orf03374 (plyE) and orf14777 (plyP) were designed and used to screen the genomic cosmid library by PCR.

\section{Genome sequencing and analysis}

Genome sequencing was accomplished by 454 sequencing technology. Open reading frames were analyzed using the Frame Plot 3.0 beta online [61], and the analysis of the deduced function of the proteins were carried out by the NCBI website [62]. Primer design, multiple nucleotide sequence alignments and analysis were performed using the BioEdit. The NRPS-PKS architecture was analyzed by NRPS-PKS online website (http://nrps. igs.umaryland.edu/nrps/) [63] and the prediction of ten amino acid of the conserved substrate-binding pocket of the A domain was performed using the online program NRPS predictor (http://ab.inf.unituebingen.de/toolbox/ index.php?view=domainpred) [64].

\section{Construction of gene inactivation mutants}

All the mutant strains in this study were generated by homologous recombination according to the standard method [65]. The target genes were replaced with an apramycin-resistance gene from pIJ773 on SuperCos1 by traditional PCR-targeting technique. Then the recombinant plasmids were transformed into E. coli S17-1 cells for conjugation. The exconjugants would appear three days later and could be transferred to a new growth medium supplemented with apramycin $(60 \mu \mathrm{g} / \mathrm{mL})$ and nalidixic acid $(100 \mu \mathrm{g} / \mathrm{mL})$. Double-crossover mutants were identified through diagnostic PCR with corresponding primers (Additional file 1: Table S3).

\section{LC-MS analyses of wild type and mutant strains}

After finishing the fermentation, the culture broth of wild type and mutant strains were extracted by equal volume of ethyl acetate. The supernatant of the ethyl acetate phase was concentrated by rotary evaporator under the reduced pressure and finally dissolved in methanol $(400 \mu \mathrm{L})$ for the LC-MS analysis using the Agilent 1100 series LC/MSD Trap system. The conditions for the LC-MS analysis are as follows: $55-100 \%$ B (linear gradient, $0-25 \mathrm{~min}$, solvent $\mathrm{A}$ is water containing $0.1 \%$ formic acid, solvent B is acetonitrile containing $0.1 \%$ formic acid), $100 \%$ B (26-30 min) at the flow rate of $0.3 \mathrm{~mL} / \mathrm{min}$ with a reverse-phase column ZORBAX SBC18 (Agilent, $5 \mu \mathrm{m}, 150 \mathrm{~mm} \times 4.6 \mathrm{~mm}$ ). Figure 4B was recorded with the conditions: $35-95 \% \mathrm{~B}$ (linear gradient, 0-20 $\mathrm{min}), 100 \% \mathrm{~B}(21-25 \mathrm{~min}), 35 \% \mathrm{~B}(25-40 \mathrm{~min})$ at the flow rate of $0.3 \mathrm{~mL} / \mathrm{min}$.

\section{Nucleotide sequence accession number}

The sequence of the polyoxypeptin A biosynthetic gene cluster was deposited in GenBank with accession number KF386858.

\section{Additional file}

Additional file 1: Electronic supplementary materials are available: bacterial strains (Table S1), plasmids (Table S2), primers (Table S3), and the substrate-specific codon sequences (Table S4); sequence alignment (Figures S1-6) and mutant construction (Schemes S1-8).

\section{Competing interests}

The authors declare that they have no competing interests.

\section{Authors' contributions}

SL designed this study; YD, YW, TH performed the experiments; YD, MT, ZD and SL analyzed data; YD and SL wrote this manuscript; MT and ZD edited this manuscript; All authors read and approved the final manuscript.

\section{Acknowledgments}

This work was financially supported by the 973 programs (2010CB833805 for SL) and (2009CB1 18901 for ZD) from MOST, the key project (311018) from MOE and NSFC (31070057 for SL; 31121064 for ZD).

\section{Author details}

'State Key Laboratory of Microbial Metabolism, School of Life Sciences and Biotechnology, Shanghai Jiao Tong University, 800 Dongchuan Road, Shanghai 200240, China. ${ }^{2}$ Current address: Department of Chemistry at the Scripps Research Institute, Jupiter, FL 33458, USA.

Received: 7 November 2013 Accepted: 4 February 2014

Published: 8 February 2014

\section{References}

1. Umezawa KNK, Uemura T, et al: Polyoxypeptin isolated from Streptomyces: a bioactive cyclic depsipeptide containing the novel amino acid 3-hydroxy-3-methylproline. Tetrahedron Lett 1998, 39(11):1389-1392.

2. Umezawa K, Nakazawa K, Ikeda Y, Naganawa H, Kondo S: Polyoxypeptins A and B produced by Streptomyces: apoptosis-inducing cyclic depsipeptides containing the novel amino acid (2S,3R)-3-hydroxy-3-methylproline. J Org Chem 1999, 64(9):3034-3038.

3. Smitka TA, Deeter JB, Hunt AH, Mertz FP, Ellis RM, Boeck LD, Yao RC: A83586C, a new depsipeptide antibiotic. J Antibiot (Tokyo) 1988, 41(6):726-733.

4. Grafe U, Schlegel R, Ritzau M, Ihn W, Dornberger K, Stengel C, Fleck WF, Gutsche W, Hartl A, Paulus EF: Aurantimycins, new depsipeptide antibiotics from Streptomyces aurantiacus IMET 43917. Production, isolation, structure elucidation, and biological activity. J Antibiot (Tokyo) 1995, 48(2):119-125.

5. Maehr H, Liu CM, Palleroni NJ, Smallheer J, Todaro L, Williams TH, Blount JF: Microbial products. VIII. Azinothricin, a novel hexadepsipeptide antibiotic. J Antibiot (Tokyo) 1986, 39(1):17-25.

6. Hayakawa Y, Nakagawa M, Toda Y, Seto H: A new depsipeptide antibiotic, citropeptin. Agric Biol Chem 1990, 54(4):1007-1011.

7. Matsumoto N, Momose I, Umekita M, Kinoshita N, Chino M, linuma H, Sawa T, Hamada M, Takeuchi T: Diperamycin, a new antimicrobial antibiotic produced by Streptomyces griseoaurantiacus MK393-AF2. I. Taxonomy, fermentation, isolation, physico-chemical properties and biological activities. J Antibiot (Tokyo) 1998, 51(12):1087-1092.

8. Maskey RP, Fotso S, Sevvana M, Uson I, Grun-Wollny I, Laatsch H: Kettapeptin: isolation, structure elucidation and activity of a new hexadepsipeptide antibiotic from a terrestrial Streptomyces sp. J Antibiot (Tokyo) 2006, 59(5):309-314.

9. Umezawa K, Ikeda Y, Naganawa H, Kondo S: Biosynthesis of the lipophilic side chain in the cyclic hexadepsipeptide antibiotic IC101. J Nat Prod 2002, 65(12):1953-1955.

10. Hensens OD, Borris RP, Koupal LR, Caldwell CG, Currie SA, Haidri AA, Homnick CF, Honeycutt SS, Lindenmayer SM, Schwartz CD, et al: L-156,602, a C5a antagonist with a novel cyclic hexadepsipeptide structure from Streptomyces sp. MA6348. Fermentation, isolation and structure determination. J Antibiot (Tokyo) 1991, 44(2):249-254. 
11. Uchihata $Y$, Ando N, Ikeda $Y$, Kondo S, Hamada M, Umezawa K: Isolation of a novel cyclic hexadepsipeptide pipalamycin from Streptomyces as an apoptosis-inducing agent. J Antibiot (Tokyo) 2002, 55(1):1-5.

12. Nakagawa M, Hayakawa $Y$, Adachi $K$, Seto H: A new depsipeptide antibiotic, variapeptin. Agric Biol Chem 1990, 54(3):791-794

13. Sakai Y, Yoshida T, Tsujita T, Ochiai K, Agatsuma T, Saitoh Y, Tanaka F, Akiyama T, Akinaga S, Mizukami T: GE3, a novel hexadepsipeptide antitumor antibiotic, produced by Streptomyces sp. I. Taxonomy, production, isolation, physico-chemical properties, and biological activities. J Antibiot (Tokyo) 1997, 50(8):659-664.

14. Agatsuma T, Sakai Y, Mizukami T, Saitoh Y: GE3, a novel hexadepsipeptide antitumor antibiotic produced by Streptomyces sp. II. Structure determination. J Antibiot (Tokyo) 1997, 50(8):704-708.

15. Tsuji RF, Yamakoshi J, Uramoto M, Koshino H, Saito M, Kikuchi M, Masuda T: Anti-inflammatory effects and specificity of L-156,602: comparison of effects on concanavalin $A$ and zymosan-induced footpad edema, and contact sensitivity response. Immunopharmacology 1995, 29(1):79-87.

16. Oelke AJ, France DJ, Hofmann T, Wuitschik G, Ley SV: Piperazic acidcontaining natural products: isolation, biological relevance and total synthesis. Nat Prod Rep 2011, 28(8):1445-1471.

17. Umezawa K, Nakazawa K, Uchihata Y, Otsuka M: Screening for inducers of apoptosis in apoptosis-resistant human carcinoma cells. Adv Enzyme Regul 1999, 39:145-156.

18. Umezawa K, Ikeda $Y$, Kawase $O$, Naganawa H, Kondo S: Biosynthesis of polyoxypeptin A: novel amino acid 3-hydroxy-3-methylproline derived from isoleucine. J Chem Soc, Perkin Trans 1(13):1550-1553.

19. Kodani S, Bicz J, Song L, Deeth RJ, Ohnishi Kameyama M, Yoshida M, Ochi K, Challis GL: Structure and biosynthesis of scabichelin, a novel trishydroxamate siderophore produced by the plant pathogen Streptomyces scabies 87.22. Org Biomol Chem 2013, 11(28):4686-4694.

20. Shen JW, Qin DG, Zhang HW, Yao ZJ: Studies on the synthesis of $(2 S, 3 R)$ 3-hydroxy-3-methylproline via C2-N bond formation. J Org Chem 2003, 68(19):7479-7484.

21. Fischbach MA, Walsh CT: Assembly-line enzymology for polyketide and nonribosomal Peptide antibiotics: logic, machinery, and mechanisms. Chem Rev 2006, 106(8):3468-3496

22. Kieser TBM, Chater KF, Butter MJ, Hopwood D: Practical Streptomyces genetics:a laboratory manual. Norwich, UK, Norwich: John Innes Foundation; 2000.

23. He Y, Sun Y, Liu T, Zhou X, Bai L, Deng Z: Cloning of separate meilingmycin biosynthesis gene clusters by use of acyltransferaseketoreductase didomain PCR amplification. App/ Environ Microbiol 2010, 76(10):3283-3292.

24. Keatinge-Clay AT: The structures of type I polyketide synthases. Nat Prod Rep 2012, 29(10):1050-1073.

25. Yadav G, Gokhale RS, Mohanty D: Computational approach for prediction of domain organization and substrate specificity of modular polyketide synthases. J Mol Biol 2003, 328(2):335-363.

26. Smith S, Tsai SC: The type I fatty acid and polyketide synthases: a tale of two megasynthases. Nat Prod Rep 2007, 24(5):1041-1072.

27. Tsai SC, Ames BD: Structural enzymology of polyketide synthases. Methods Enzymol 2009, 459:17-47.

28. Erb TJ, Berg IA, Brecht V, Muller M, Fuchs G, Alber BE: Synthesis of C5-dicarboxylic acids from C2-units involving crotonyl-CoA carboxylase/ reductase: the ethylmalonyl-CoA pathway. Proc Natl Acad Sci USA 2007, 104(25):10631-10636.

29. Erb TJ, Brecht V, Fuchs G, Muller M, Alber BE: Carboxylation mechanism and stereochemistry of crotonyl-CoA carboxylase/reductase, a carboxylating enoyl-thioester reductase. Proc Natl Acad Sci USA 2009, 106(22):8871-8876

30. Eustaquio AS, McGlinchey RP, Liu Y, Hazzard C, Beer LL, Florova G, Alhamadsheh MM, Lechner A, Kale AJ, Kobayashi Y, et al: Biosynthesis of the salinosporamide $A$ polyketide synthase substrate chloroethylmalonyl-coenzyme A from S-adenosyl-L-methionine. Proc Natl Acad Sci USA 2009, 106(30):12295-12300.

31. Quade N, Huo L, Rachid S, Heinz DW, Muller R: Unusual carbon fixation gives rise to diverse polyketide extender units. Nat Chem Biol 2012, 8(1):117-124.

32. Rachid S, Huo L, Herrmann J, Stadler M, Kopcke B, Bitzer J, Muller R: Mining the cinnabaramide biosynthetic pathway to generate novel proteasome inhibitors. Chembiochem 2011, 12(6):922-931.
33. Buntin $\mathrm{K}$, Irschik H, Weissman KJ, Luxenburger $\mathrm{E}$, Blocker $\mathrm{H}$, Muller R: Biosynthesis of thuggacins in myxobacteria: comparative cluster analysis reveals basis for natural product structural diversity. Chem Bio/ 2010, 17(4):342-356

34. Qu X, Jiang N, Xu F, Shao L, Tang G, Wilkinson B, Liu W: Cloning, sequencing and characterization of the biosynthetic gene cluster of sanglifehrin A, a potent cyclophilin inhibitor. Mol Biosyst 2011, 7(3):852-861.

35. Xu Z, Ding L, Hertweck C: A branched extender unit shared between two orthogonal polyketide pathways in an endophyte. Angew Chem Int Ed Engl 2011, 50(20):4667-4670.

36. Wilson MC, Nam SJ, Gulder TA, Kauffman CA, Jensen PR, Fenical W, Moore BS: Structure and biosynthesis of the marine streptomycete ansamycin ansalactam $A$ and its distinctive branched chain polyketide extender unit. J Am Chem Soc 2011, 133(6):1971-1977.

37. Neumann CS, Jiang W, Heemstra JR Jr, Gontang EA, Kolter R, Walsh CT: Biosynthesis of piperazic acid via N5-hydroxy-ornithine in Kutzneria spp. 744. Chembiochem 2012, 13(7):972-976

38. Fujimori DG, Hrvatin S, Neumann CS, Strieker M, Marahiel MA, Walsh CT: Cloning and characterization of the biosynthetic gene cluster for kutznerides. Proc Natl Acad Sci USA 2007, 104(42):16498-16503.

39. Ma J, Wang Z, Huang H, Luo M, Zuo D, Wang B, Sun A, Cheng YQ, Zhang $C$, Ju J: Biosynthesis of himastatin: assembly line and characterization of three cytochrome P450 enzymes involved in the post-tailoring oxidative steps. Angew Chem Int Ed Engl 2011, 50(34):7797-7802.

40. Stachelhaus T, Mootz HD, Marahiel MA: The specificity-conferring code of adenylation domains in nonribosomal peptide synthetases. Chem Biol 1999, 6(8):493-505.

41. Challis GL, Ravel J, Townsend CA: Predictive, structure-based model of amino acid recognition by nonribosomal peptide synthetase adenylation domains. Chem Biol 2000, 7(3):211-224.

42. Martin JL, McMillan FM: SAM (dependent) I AM: the S adenosylmethionine-dependent methyltransferase fold. Curr Opin Struct Biol 2002, 12(6):783-793.

43. von Dohren $\mathrm{H}$, Keller U, Vater J, Zocher R: Multifunctional Peptide Synthetases. Chem Rev 1997, 97(7):2675-2706.

44. Li W, Rokni-Zadeh H, De Vleeschouwer M, Ghequire MG, Sinnaeve D, Xie GL, Rozenski J, Madder A, Martins JC, De Mot R: The antimicrobial compound xantholysin defines a new group of Pseudomonas cyclic lipopeptides. PLoS One 2013, 8(5):e62946.

45. Claxton HB, Akey DL, Silver MK, Admiraal SJ, Smith JL: Structure and functional analysis of RifR, the type II thioesterase from the rifamycin biosynthetic pathway. J Biol Chem 2009, 284(8):5021-5029.

46. Koglin A, Lohr F, Bernhard F, Rogov W, Frueh DP, Strieter ER, Mofid MR, Guntert $P$, Wagner $G$, Walsh $C T$, et al: Structural basis for the selectivity of the external thioesterase of the surfactin synthetase. Nature 2008, 454(7206):907-911.

47. Schwarzer D, Mootz HD, Linne U, Marahiel MA: Regeneration of misprimed nonribosomal peptide synthetases by type II thioesterases. Proc Natl Acad Sci USA 2002, 99(22):14083-14088.

48. Lin S, Huang T, Shen B: Tailoring enzymes acting on carrier proteintethered substrates in natural product biosynthesis. Methods Enzymol 2012, 516:321-343.

49. Thomas MG, Burkart MD, Walsh CT: Conversion of L-proline to pyrrolyl-2carboxyl-S-PCP during undecylprodigiosin and pyoluteorin biosynthesis. Chem Biol 2002, 9(2):171-184.

50. Pohlmann V, Marahiel MA: Delta-amino group hydroxylation of L-ornithine during coelichelin biosynthesis. Org Biomol Chem 2008 6(10):1843-1848.

51. Jiang W, Cacho RA, Chiou G, Garg NK, Tang Y, Walsh CT: EcdGHK are three tailoring iron oxygenases for amino acid building blocks of the echinocandin scaffold. J Am Chem Soc 2013, 135(11):4457-4466.

52. Shimada N, Morimoto K, Naganawa H, Takita T, Hamada M, Maeda K, Takeuchi T, Umezawa H: Antrimycin, a new peptide antibiotic. J Antibiot (Tokyo) 1981, 34(12):1613-1614

53. Umezawa K, Ikeda Y, Uchihata Y, Naganawa H, Kondo S: Chloptosin, an apoptosis-inducing dimeric cyclohexapeptide produced by Streptomyces. J Org Chem 2000, 65(2):459-463.

54. Fox KR, Davies H, Adams GR, Portugal J, Waring MJ: Sequence-specific binding of luzopeptin to DNA. Nucleic Acids Res 1988, 16(6):2489-2507. 
55. Lingham RB, Hsu AH, O'Brien JA, Sigmund JM, Sanchez M, Gagliardi MM, Heimbuch BK, Genilloud O, Martin I, Diez MT, et al: Quinoxapeptins: novel chromodepsipeptide inhibitors of HIV-1 and HIV-2 reverse transcriptase. I. The producing organism and biological activity. J Antibiot (Tokyo) 1996, 49(3):253-259.

56. Huang X, Roemer E, Sattler I, Moellmann U, Christner A, Grabley S: Lydiamycins A-D: cyclodepsipetides with antimycobacterial properties. Angew Chem Int Ed Engl 2006, 45(19):3067-3072.

57. Miller ED, Kauffman CA, Jensen PR, Fenical W: Piperazimycins: cytotoxic hexadepsipeptides from a marine-derived bacterium of the genus Streptomyces. J Org Chem 2007, 72(2):323-330.

58. Fehr T, Kallen J, Oberer L, Sanglier JJ, Schilling W: Sanglifehrins A, B, C and $\mathrm{D}$, novel cyclophilin-binding compounds isolated from Streptomyces sp. A92-308110. II. Structure elucidation, stereochemistry and physicochemical properties. J Antibiot (Tokyo) 1999, 52(5):474-479.

59. Zhang H, Chen J, Wang H, Xie Y, Ju J, Yan Y: Structural analysis of HmtT and $\mathrm{HmtN}$ involved in the tailoring steps of himastatin biosynthesis. FEBS Lett 2013, 587(11):1675-1680.

60. Huang T, Wang Y, Yin J, Du Y, Tao M, Xu J, Chen W, Lin S, Deng Z Identification and characterization of the pyridomycin biosynthetic gene cluster of Streptomyces pyridomyceticus NRRL B-2517. J Biol Chem 2011, 286(23):20648-20657.

61. Ishikawa J, Hotta K: FramePlot: a new implementation of the frame analysis for predicting protein-coding regions in bacterial DNA with a high $\mathrm{G}+\mathrm{C}$ content. FEMS Microbiol Lett 1999, 174(2):251-253.

62. Altschul SF, Madden TL, Schaffer AA, Zhang J, Zhang Z, Miller W, Lipman DJ: Gapped BLAST and PSI-BLAST: a new generation of protein database search programs. Nucleic Acids Res 1997, 25(17):3389-3402

63. Ansari MZ, Yadav G, Gokhale RS, Mohanty D: NRPS-PKS: a knowledgebased resource for analysis of NRPS/PKS megasynthases. Nucleic Acids Res 2004, 32(Web Server issue):W405-W413.

64. Rausch C, Weber T, Kohlbacher O, Wohlleben W, Huson DH: Specificity prediction of adenylation domains in nonribosomal peptide synthetases (NRPS) using transductive support vector machines (TSVMs). Nucleic Acids Res 2005, 33(18):5799-5808.

65. Gust BKT, Chater K: PCR targeting system in Streptomyces coelicolor A3(2). Norwich U K: The John Innes Foundation; 2002.

doi:10.1186/1471-2180-14-30

Cite this article as: Du et al:: Identification and characterization of the biosynthetic gene cluster of polyoxypeptin $\mathrm{A}$, a potent apoptosis inducer. BMC Microbiology 2014 14:30.

\section{Submit your next manuscript to BioMed Central and take full advantage of:}

- Convenient online submission

- Thorough peer review

- No space constraints or color figure charges

- Immediate publication on acceptance

- Inclusion in PubMed, CAS, Scopus and Google Scholar

- Research which is freely available for redistribution 